\title{
Association between long non-coding RNAs expression and pathogenesis and progression of gliomas (Review)
}

\author{
JIE ZHOU, WEI XIANG, SHENJIE LI, QI HU, TAO PENG, LIGANG CHEN and YANG MING \\ Department of Neurosurgery, The Affiliated Hospital of Southwest Medical University, \\ Southwest Medical University, Luzhou, Sichuan 646000, P.R. China
}

Received July 26, 2017; Accepted December 13, 2017

DOI: $10.3892 / \mathrm{ol} .2018 .7875$

\begin{abstract}
The incidence rate of gliomas is the highest among primary brain tumors. Although the understanding of the molecular pathology of glioma has improved during the previous two decades, effective therapies are not yet available to treat these tumors. Previous studies have indicated that long non-coding RNAs (lncRNAs) have a close association with glioma, suggesting that lncRNAs may be potential targets for the development of novel treatments for glioma. The present review summarized the latest studies on the dysregulation of lncRNAs in glioma, and discussed their potential use in the diagnosis, prognosis and therapies of glioma. The emergence of lncRNAs has revealed an additional facet to glioma oncogenesis. An improved understanding of their functions is important to advance lncRNA-based diagnosis, prognosis and therapeutic interventions of glioma.
\end{abstract}

Correspondence to: Dr Ligang Chen or Dr Yang Ming, Department of Neurosurgery, The Affiliated Hospital of Southwest Medical University, Southwest Medical University, 25 Taiping Street, Luzhou, Sichuan 646000, P.R. China

E-mail: chengligang.cool@163.com

E-mail: zj000718@yeah.net

Abbreviations: CASC2, cancer susceptibility candidate 2; CNS, central nervous system; CRNDE, colorectal neoplasia differentially expressed; ceRNA, competing endogenous RNA; DNMT1, DNA methyltransferase 1; ERL, erlotinib; HOTAIR, HOX transcript antisense RNA; lncRNAs, long non-coding RNAs; MEG3, maternally expressed gene 3; MALAT1, metastasis associated lung adenocarcinoma transcript 1; miRNA, microRNA; ncRNA, non-coding RNA; piRNA, PIWI-interacting RNAs; rRNA, ribosomal RNA; siRNA, small interfering RNA; snRNA, small nuclear RNA; snoRNA, small nucleolar RNA; tRNA, transfer RNA; TMZ, temozolomide

Key words: biomarkers, function, glioma, long non-coding RNAs, therapeutic targets

\section{Contents}

1. Introduction

2. Classification and characteristics of lncRNAs

3. Dysregulation of IncRNAs in gliomas

4. Clinical applications in gliomas

5. Conclusion and future perspectives

\section{Introduction}

Previous studies have identified that the human genome contains $\sim 21,000$ genes, and only $<2 \%$ of them are protein-coding genes $(1,2)$. In the previous decades, studies of protein-coding genes have led to improved understanding of their participation in tumorigenesis and tumor characteristics, consequentially establishing a number of protein prognostic markers and therapeutic targets in numerous types of cancer (3-6). Furthermore, larger numbers of non-coding RNAs (ncRNAs), including microRNAs (miRNAs), PIWI-interacting RNAs (piRNAs), small interfering RNAs (siRNAs), small nuclear RNAs (snRNAs), small nucleolar RNAs (snoRNAs) and lncRNAs are expressed at lower levels to fulfill regulatory functions to control complex physiopathological processes in humans $(7,8)$. Therefore, it is important to characterize the functions of the large majority of ncRNAs.

NcRNAs may be classified by their biological functions: housekeeping ncRNAs and regulatory ncRNAs. Housekeeping ncRNAs are usually expressed constitutively, including ribosomal RNA (rRNAs), snRNAs, snoRNAs and transfer RNAs (tRNAs). Regulatory ncRNAs, according to their length, comprise short regulatory ncRNAs, including miRNAs, siRNAs and piRNAs and long regulatory ncRNAs (8-10).

LncRNAs are a class of ncRNAs with $>200$ nucleotides in length. It is now recognized that IncRNAs function as key regulatory players in a number of biological processes, including embryonic development, cellular differentiation and cancer (11). IncRNAs regulate their target genes at transcriptional or post-transcriptional levels. Previously, the dysregulation of IncRNAs has been closely associated with carcinogenesis and cancer progression. Compared with the protein-coding genes, lncRNAs exhibit more tissue- and time-specific expression patterns, and their expressions are more closely associated with their biological function and 
tumor status, indicating enormous potential roles of lncRNAs as diagnostic and prognostic biomarkers, and as therapeutic targets in cancer $(3,12-14)$. For example, the variant genotypes of rs7763881 in the hepatocellular carcinoma up-regulated long non-coding RNA gene may be responsible for the decreased susceptibility to hepatitis B virus-associated carcinogenesis in liver, suggesting that genetic variations in lncRNAs are associated with cancer susceptibility (15). In addition, aberrant expression of lncRNAs has been employed in cancer diagnosis and monitoring (16). H19 is upregulated in the plasma of patients with gastric cancer, and its expression enabled the differentiation of early stage gastric cancer from healthy controls (17). Subsequent studies have indicated that the level of H19 may be used to monitor and reflect the tumor dynamics in patients with gastric cancer (18). Furthermore, lncRNA expression profiles may also be used to identify clinically relevant cancer subtypes that predict tumor biological behavior, therapeutic responsiveness and clinical prognosis (19-23).

Gliomas represent $31 \%$ of all central nervous system (CNS) tumors diagnosed in the United States, and $81 \%$ of all malignant CNS tumor types with high morbidity and mortality (2006-2010) (24). Despite the treatment options of surgical resection followed by radiotherapy and chemotherapy, the overall survival times of patients with glioma, particularly patients with malignant glioma, were low. The understanding of the genetic and molecular makeup of gliomas has been advanced during the previous the decades. However, there remains a lack of effective therapies for these tumors. Therefore, an improved understanding of glioma pathogenesis is urgently required. Previous studies suggest that lncRNAs have a close association with glioma, but their roles and the underlying mechanisms remain elusive. In the present review, the recent progress on lncRNAs in the development of glioma was summarized, and their possible functions and pathogenesis mechanisms in regulating biological behaviors of glioma were discussed.

\section{Classification and characteristics of IncRNAs}

LncRNAs are a large and heterogeneous group of RNAs, reflecting indirectly their enormous variety and structural complexity. Based on its genomic location to protein-coding genes, an lncRNA may be placed broadly into several categories: i) bidirectional; ii) enhancer; iii) intergenic; iv) intronic; v) sense and vi) anti-sense lncRNAs. The expression of bidirectional $\operatorname{lncRNAs}$ is initiated within the vicinity $(>1 \mathrm{~kb})$ of a neighboring coding transcript of the opposite strand. Enhancer lncRNAs are located in the enhancer regions of the promoter of a coding transcript. Intergenic lncRNAs are transcribed from regions between two coding transcripts. Intronic lncRNAs are derived entirely from within the introns of a coding transcript. Sense lncRNAs overlap with a part of or the entire sense strand of a transcript. Anti-sense lncRNAs are transcribed from the anti-sense direction to the transcripts of a gene (Fig. 1) $(25,26)$.

The ways in which IncRNAs regulate gene expression can also be grouped into three categories, which include transcriptional and post-transcription regulation, and other mechanisms. Transcriptional regulation is where lncRNAs regulate gene expression through transcriptional interference and chromatin remodeling (27). Post-transcription regulation involves the regulation of RNA splicing by modulating the functions of splicing factors or by directly binding to pre-mRNA sequences. LncRNAs may also block translation through interaction with translation factors or ribosomes (28-30). Other mechanisms of gene expression by lncRNAs include protein localization, telomere replication and RNA interference $(31,32)$.

LncRNAs may also be classified into four archetypes based on the molecular mechanisms of their functions: i) signal: lncRNAs may serve as molecular signals for gene regulation; ii) decoy: IncRNAs act as 'molecular sinks' that bind and sequestrate protein targets but do not exert any additional functions; iii) guide: IncRNAs interact with proteins and guide the localization of ribonucleoprotein complexes to specific targets; iv) scaffold: IncRNAs function as central platforms for multiple molecules to form scaffolding complexes to regulate their functions (33).

\section{Dysregulation of IncRNAs in gliomas}

Previous studies have suggested that several lncRNAs are involved in the development of gliomas and associated with various biological behaviors of tumors, including proliferation, migration, invasion and apoptosis. The lncRNAs that are associated with gliomas are summarized in Table I. In the following section, the potential roles of several lncRNAs in the development of glioma, and their potential for clinical applications for glioma treatment, are discussed.

\section{Oncogenic lncRNAs}

H19. IncRNA H19, a paternally imprinted gene residing close to the telomeric region of chromosome $11 \mathrm{p} 15.5$, was first identified as a tumor suppressor $(34,35)$. However, subsequent studies indicated that the function of H19 was tissue and developmental stage specific. H19 is oncogenic in thyroid cancer, hepatocellular and bladder carcinoma (36-38).

In gliomas, H19 contributes to tumorigenesis and tumor progression via several mechanisms. Jiang et al (39) identified that the increased expression of H19 IncRNA promoted the invasion and angiogenesis of glioblastoma cells in culture and increased the rate of growth of xenograft tumors in mice. Jia et al (40) revealed that H19, as a molecular sponge, promoted glioma-induced angiogenesis by downregulating miRNA-29a. Chen et al (41) demonstrated that H19 was upregulated in recurrent gliomas compared with primary gliomas, suggesting that it was associated the development of glioma. Shi et al (42) identified that H19 expression associated with tumor grade, that $\mathrm{H} 19$ promoted glioma progression via the H19-derived miR-675/CDH13 pathway, and that the suppression of H19 expression inhibited the invasion of glioma cells. Furthermore, H19 was expressed at high levels in the embryo and was hypothesized to serve an important role in the maintenance of the stemness in hematopoietic/embryonic stem cells (42-45). A previous study has demonstrated that $\mathrm{H} 19$ is upregulated in $\mathrm{CD}_{133^{+}}$glioblastoma cells compared with CD133- tumor cells. The overexpression of H19 in CD133 tumor cells promoted tumor growth, indicating the importance of H19 in promoting stemness of glioblastoma cells (46). Li et al (47) reported that the knockdown of H19 was able to significantly reduce the expression of stem cell markers. A high expression of H19 was considered to transform normal astrocytes into glioma stem cells, suggesting that H19 may 


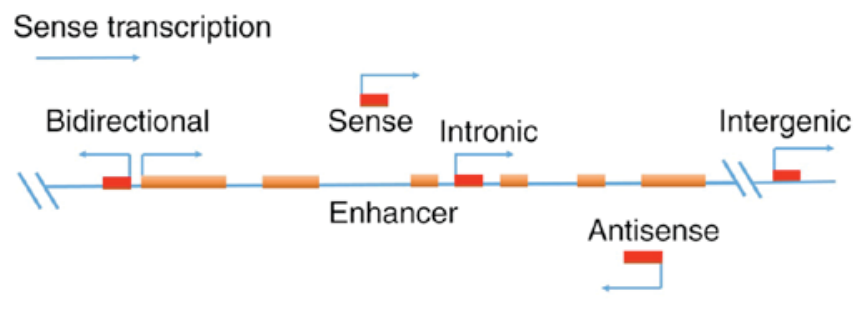

- Protein-coding gene $=$ Long non-coding gene

Figure 1. Long non-coding RNAs may be classified as bidirectional, enhancer, intergenic, intronic, sense or antisense based on their genomic location to neighboring protein-coding genes.

have role in contributing to the malignancy and stemness of glioblastoma cells. In addition, aberrant expression of H19 was observed in tumors from patients with temozolomide (TMZ) resistance, and TMZ-resistant cell lines (48). The silencing of H19 may regulate drug resistance genes, including multidrug resistance protein 1 , multidrug resistant associated protein 1 and ATP-binding cassette subfamily G member 2; and may promote apoptosis in sensitized tumor cells in drug-resistant glioma (48). Furthermore, Jiang et al (39) also demonstrated that the stable overexpression of H19 in U87MG and U373MG cell lines promoted tumor formation and induced tumor cell proliferation and angiogenesis in an in vivo murine xenograft model.

Metastasis-associated lung adenocarcinoma transcript 1 (MALAT1). MALAT1, an 1ncRNA with a length of $8.7 \mathrm{~kb}$, is located on chromosome 11q13.1 (49). MALAT1 was originally identified as an lncRNA that is overexpressed in metastatic non-small cell lung cancer, and it is considered to be a potential therapeutic target for non-small cell lung cancer (50). The expression of MALAT1 is dynamically regulated to promote the development of various types of cancer, including ovarian, pancreatic, lung and cervical cancer (51-54). To date, MALAT1 has been reported to serve a pro-oncogenic role in the progression of gliomas. Ma et al (55) revealed that MALAT1 expression was associated with the malignant status of glioma and that high levels of MALAT1 expression were associated with poor prognosis in patients with glioma. Xiang et al (56) identified that the level of MALAT1 was increased in tumor tissues compared with normal brain tissues in glioma, and the knockdown of MALAT1 resulted in the downregulation of cyclin D1 and MYC, the inhibition of tumor growth and induction of cell apoptosis in gliomas. Vassallo et al (57) identified that MALAT1 was downregulated by WNT inhibitory factor 1 via the Wnt family member 5A/tumor protein 38-mitogen-activated protein kinase (MAPK)/Ca ${ }^{2+}$ non-canonical WNT signaling axis, which led to an inhibition of migration and invasion of glioma cells. Ma et al (58) demonstrated that MALAT1 was upregulated in tumor endothelial cells compared with normal endothelial cells in glioma. Functional experiments indicated that MALAT1 acted as a competing endogenous RNA (ceRNA), which may interact with miR-140 to increase the permeability of the blood-tumor barrier. By contrast, Han et al (59) reported that MALAT1 served as a tumor suppressor gene in glioma. Han et al (59) demonstrated that the overexpression of MALAT1 caused a significant reduction in cell proliferation and invasion by inactivating the extracellular signal-related kinase/MAPK signaling pathway in vitro, and in tumorigenicity in subcutaneous and intracranial human glioma xenograft models. Nucleotide sequences of MALAT1 are highly conserved throughout evolution, suggesting that it has an important cellular function (49). However, MALAT1 may not a good therapeutic candidate due to its high basal level of expression in normal brains. Therefore, additional studies are required to investigate the role of MALAT1 and its target genes in glioma.

HOX transcript antisense RNA (HOTAIR). HOTAIR, an lncRNA of $>2,100$-nucleotides in length, is transcribed from the antisense of the HOXC gene locus in chromosome 12 (60). It has been demonstrated that the overexpression of HOTAIR is associated with proliferation, invasion and chemoresistance of tumor cells. Therefore, HOTAIR is considered to be a poor prognostic factor in various types of cancer, including hepatocellular carcinoma, gastric and lung cancer $(61,62)$. HOTAIR has been investigated as an important marker for molecular subtypes in glioma, which may serve as a potential therapeutic target for classical and mesenchymal gliomas (63). Zhou et al (64) reported that the expression of HOTAIR was associated with overall survival in patients with glioblastoma. Additionally, cell cycle arrest and attenuation of invasion in glioblastoma cells may be induced by HOTAIR depletion and subsequent inhibition of Nemo-like kinase/ $\beta$-catenin axis. Similarly, Fang et al (65) suggested that the inhibition of HOTAIR by superparamagnetic iron oxide nanoparticles mediated siRNA transfection-induced programmed cell death 4 expression, which suppressed the proliferation, invasion and tumorigenicity of glioma stem cells. Recently, accumulating evidence has suggested that the reciprocal association between miRNA and IncRNA is actively involved in cancer pathogenesis (66). Ke et al (67) demonstrated that HOTAIR was significantly upregulated in glioma tissues and cell lines compared with normal controls. Furthermore, it was suggested that the knockdown of HOTAIR may lead to the inhibition of FGF1 by upregulating miR-326, which suppressed tumor growth in vitro and in vivo. Yang et al (68) also confirmed that the survival time of nude mice was extended in a short hairpin-HOTAIR group compared with that of control groups. A recent study indicated that HOTAIR, acting as an endogenous 'sponge', may bind with miR-141 to regulate the epigenetic modification of the miRNA-induced repression of spindle and kinetochore associated complex subunit 2 to promote the proliferation and invasion of glioma cells (69). Additionally, Wang et al (70) also demonstrated that miR-148b-3p may inhibit glioma cell growth by directly downregulating HOTAIR. These data suggest that the inhibition of HOTAIR activity may potentially be used as a novel therapy for the treatment of glioma.

Colorectal neoplasia differentially expressed (CRNDE). CRNDE, which is transcribed from the strand opposite to the adjacent iroquois homeobox 5 gene in chromosome 16, was initially regarded as a pro-oncogenic lncRNA that is upregulated in colorectal cancer (71). CRNDE serves a vital role in the development of numerous organs including breast, skin, and bronchial epithelium. Notably, an increased expression 


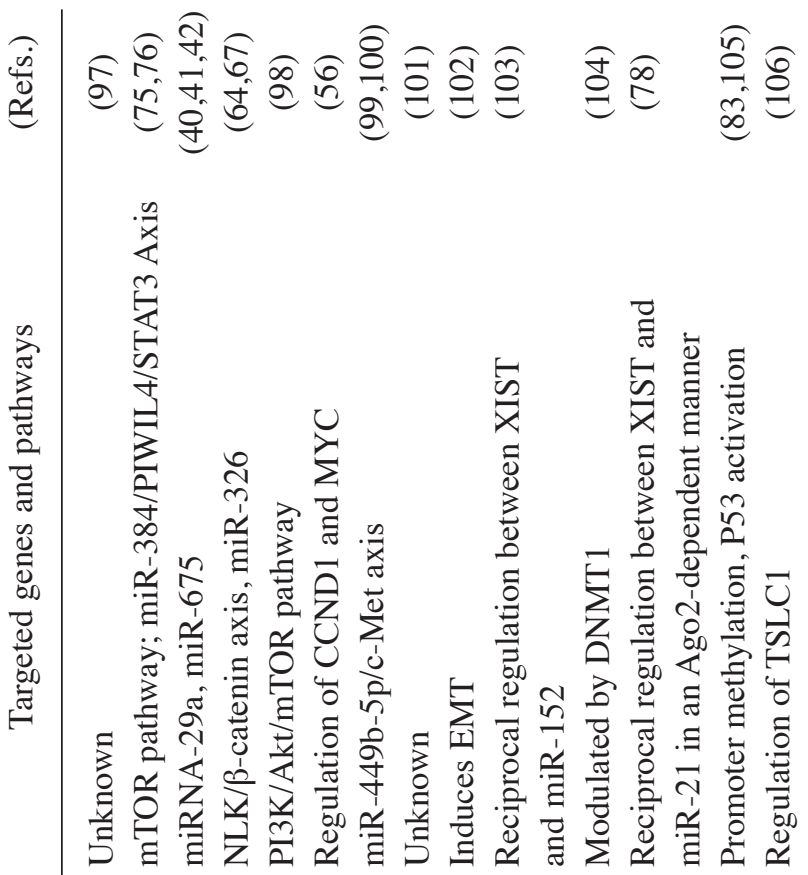

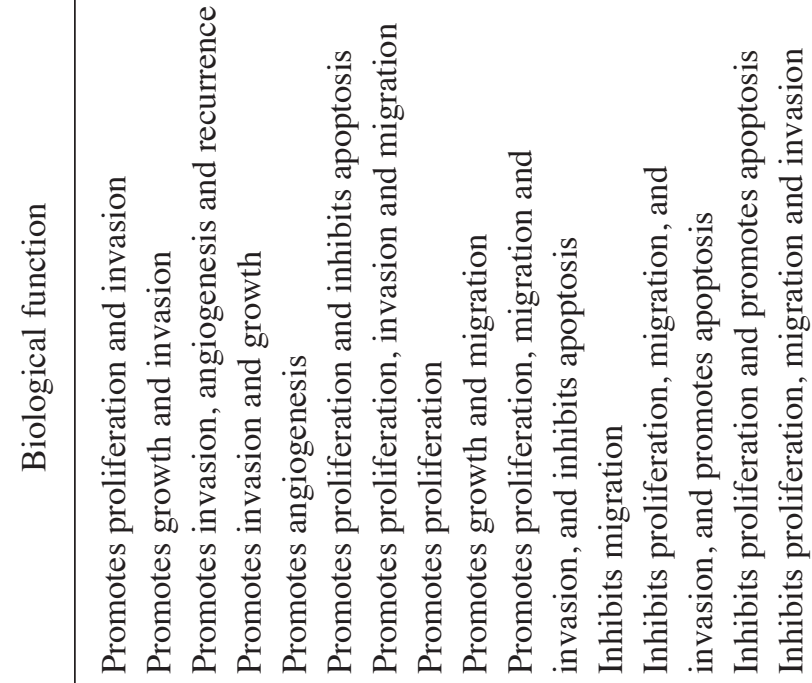

$\frac{n}{2}$

至

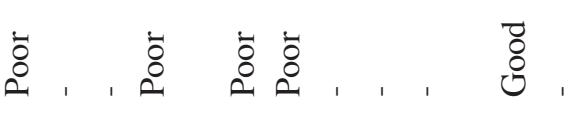

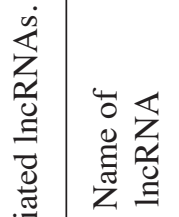

$\Xi £$

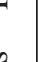

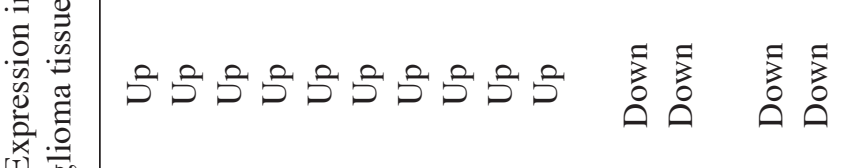

ค ค ค

(2)

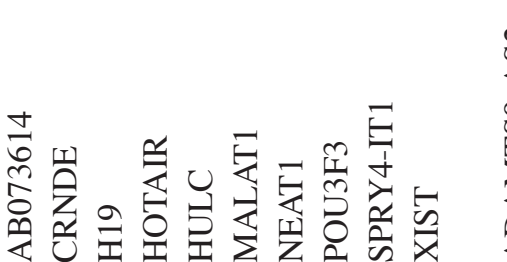

究

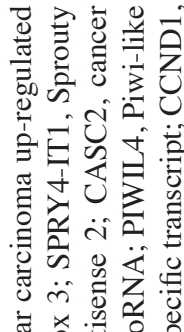

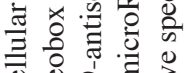

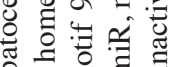

ॠथ

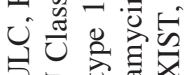

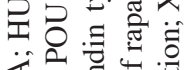

乙一ं

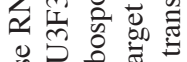

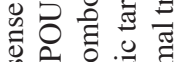

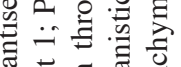

늠등

:

要

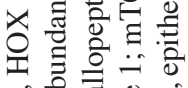

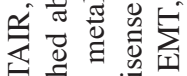

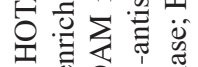

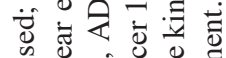

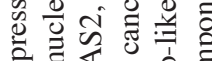

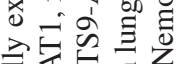

焉罗吉艺

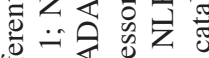

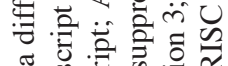

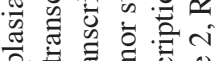

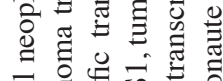

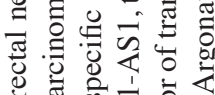

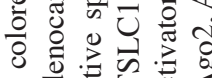

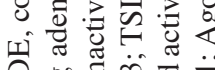

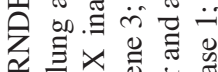

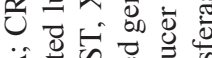

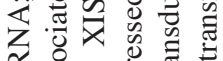

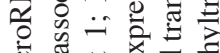

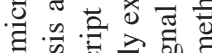

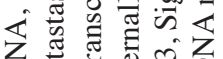

过氙它

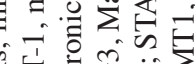

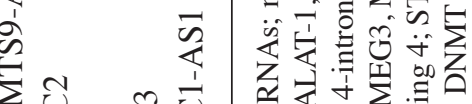

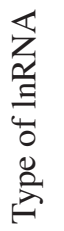

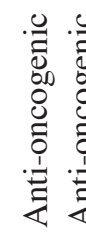

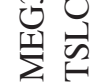

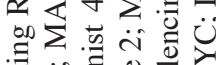

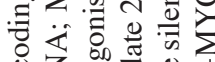

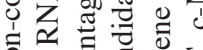

范

on

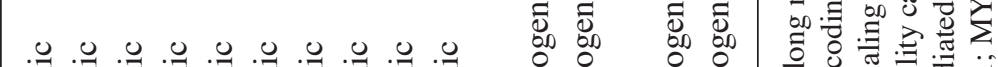

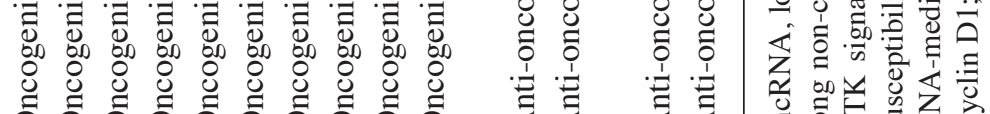




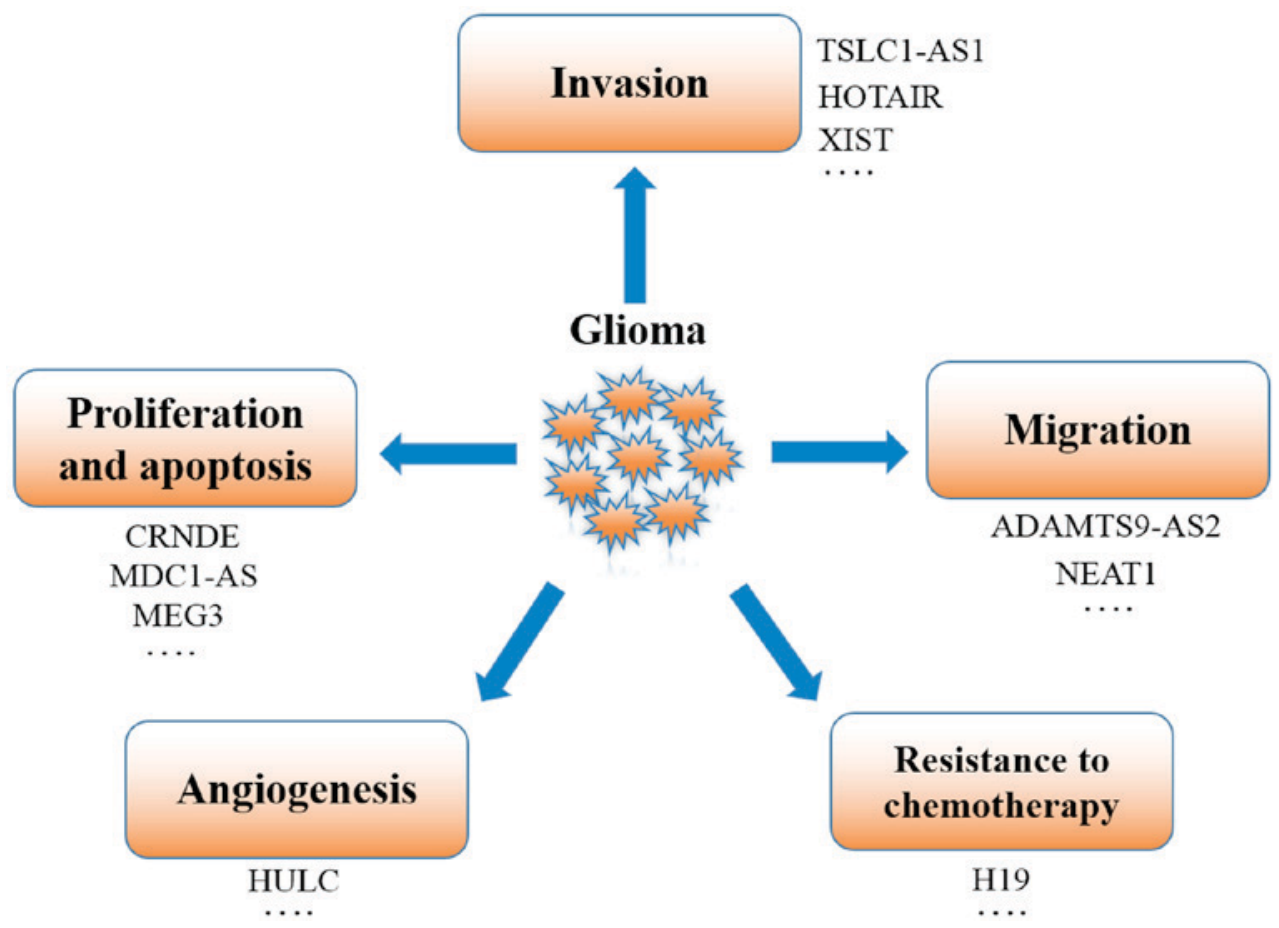

Figure 2. Long non-coding RNAs mediate various biological processes to regulate the progression of glioma. Certain representative lncRNAs function as oncogenes or tumor suppressors in specific biological process of glioma. TSLC1-AS1, tumor suppressor in lung cancer 1-antisense 1; HOTAIR, HOX transcript antisense RNA; XIST, X inactive specific transcript; CRNDE, colorectal neoplasia differentially expressed; MDC1-AS, Mediator of DNA damage checkpoint 1-antisense; MEG3, Maternally expressed gene 3; HULC, Hepatocellular carcinoma up-regulated long non-coding RNA; ADAMTS9-AS2, ADAM metallopeptidase with thrombospondin type 1 motif 9-antisense 2; NEAT1, nuclear enriched abundant transcript 1.

of CRNDE has been identified in a variety of solid tumors, including brain tumors (72). Previous studies indicated that the expression of CRNDE was markedly increased in primary and recurrent gliomas (73). Zhang et al (74) identified that CRNDE was significantly overexpressed in glioma tissues, and that the expression level of CRNDE was positively associated with the pathological grades of glioma. Similarly, Zheng et al (75) demonstrated that CRNDE promoted migration, invasion and proliferation, and inhibited apoptosis in glioma cells through regulating the expression levels of the miR-384/Piwi-like RNA-mediated gene silencing 4/signal transducer and activator of transcription 3 axis. Consistent with these data, Wang et al (76) demonstrated that CRNDE was upregulated lncRNA in glioma compared with normal tissues in their study, and that the overexpression of CRNDE promoted proliferation and invasion in glioma through the mechanistic target of rapamycin pathway in vitro and in vivo. These results suggest that understanding the underlying mechanisms whereby CRNDE functions in glioma may reveal a novel therapeutic strategy for the treatment of glioma in future.

\section{Tumor-suppressive lncRNAs}

Cancer susceptibility candidate 2 (CASC2). CASC2 has been identified as a tumor suppressor in numerous types of solid tumors (77). The role of CASC2 in glioma pathogenesis has been examined by several studies. Wang et al (77) indicated that a low level of CASC2 expression was detected in gliomas. The findings of Wang et al (78) suggested that CASC2 served as a tumor suppressor role via regulation of miR-21 in an Ago2-dependent manner in gliomas. Furthermore, a study by Liao et al (79) demonstrated that the low expression of CASC2 was associated with malignant characteristics and poor clinical prognosis in glioma. The overexpression of CACS2 inhibits the proliferation of glioma cells and amplifies TMZ-induced repression of cell proliferation through the direct inhibition of miR-181a. However, whether CACS2 has the same effect in vivo has not been reported in glioma. Therefore, the role of CACS2 in a mouse model of glioma requires additional investigation.

Maternally expressed gene 3 (MEG3). MEG3 is a maternal imprinting gene at the delta like non-canonical notch ligand 1-MEG3 locus on chromosome 14q32.3 in humans (80). A number of previous studies demonstrated that MEG3 was expressed in a number of normal tissues, with particularly marked expression in the brain, but absent or low expression in multiple types of tumors, including cervical carcinoma, breast adenocarcinoma, meningioma and glioma $(81,82)$. Wang et al (83) demonstrated that the expression of MEG3 was decreased in tumor tissues compared with adjacent non-tumor tissues in gliomas. Furthermore, ectopic expression of MEG3 inhibited the growth of glioma cells by activation of the p53 signaling pathway. Liu et al (84) also suggested that MEG3 served an important role in genotoxic stress-induced glioma cell death. Similarly, Li et al (84) indicated that a low level of MEG3 expression was observed in glioma tissues. This low expression of MEG3 was due to DNA methyltransferase 1 (DNMT1), which is mediated by hypermethylation of the MEG3 promoter. Furthermore, the inhibition of DNMT1 repressed the growth and resulted in apoptosis of glioma cells in a p53-dependent manner. Additionally, Zhang et al (85) also demonstrated that MEG3 markedly reduced tumor volume and 
the expression of $\mathrm{Ki}-67$ and proliferating cell nuclear antigen in vivo.

\section{Clinical applications in gliomas}

With an improved understanding of their functions, lncRNAs are becoming an important focus of study as a novel type of cancer biomarker for diagnosis, treatment and prognostic prediction. At present, certain lncRNAs have been examined for their potential clinical applications. It has been demonstrated that specific lncRNA profiles are associated with tumor subtypes, mutational status and the survival time of patients with glioma, based on the analysis of RNA-seq datasets from The Cancer Genome Atlas (86). Gliomas with oxalosuccinate decarboxylase (IDH1) mutations exhibited a unique lncRNA gene expression signature that was different from that of tumors exhibiting the wild-type IDH1 gene (87). As prognostic markers, the four lncRNAs (AGAP2-AS1, TPT1-AS1, LINC01198 and MIR155HG) were suggested to have prognostic value for patients with anaplastic gliomas (88). Furthermore, IncRNAs have demonstrated potential in applications for non-invasive detection of cancer. Zhou et al (18) identified that the level of $\mathrm{H} 19$ in the plasma of patients with gastric cancer was significantly increased compared with healthy controls. In addition, the plasma level of H19 was decreased markedly in postoperative patients compared with that in preoperative patients. The plasma level of H19 may be used as a non-invasive method to evaluate glioma progression in the future.

lncRNAs, due to their highly tissue-specific expression in cancer phenotypes, are potential targets for cancer therapy. Preclinical studies have demonstrated the therapeutic efficacy of antisense oligonucleotides targeting cancer-associated lncRNAs, including MALAT-1 and H19 (89,90). At present, certain lncRNAs have been identified to be associated with glioma therapy. Amit et al (91) revealed that a construct expressing the diphtheria toxin A-fragment, under the control of $\mathrm{H} 19$ and insulin-like growth factor 2 P4 promoters, demonstrated anti-tumoral efficacy against glioblastoma in vitro and in vivo. The BET bromodomain inhibitor, I-BET151 inhibited the growth of glioblastoma cells in vitro and in vivo by directly reducing HOTAIR expression (92). Similarly, Ke et al (67) also reported that HOTAIR knockdown inhibited tumor growth. In addition, aberrant expression of growth arrest specific transcript 5 (GAS5) lncRNA has also been associated with chemoresistance in glioma. García-Claver et al (93) revealed that the GAS5 lncRNA was markedly upregulated following treatment with erlotinib (ERL) in ERL-sensitive and -resistant glioma. The knockdown of GAS5 sensitized U87MG cells to ERL treatment. Similarly, in tumor tissues and cell lines from patients with TMZ resistance, H19 was significantly upregulated (48). The silencing of H19 may decrease the half-maximal inhibitory concentration values for TMZ, and increase the apoptotic rate of glioma cells (48). In summary, these results provide experimental basis for the use of lncRNAs as novel therapeutic targets in gliomas. IncRNA-based therapeutics may represent a novel direction for the treatment of glioma, although studies concerning their safety, efficacy and more efficient delivery systems are required.

\section{Conclusion and future perspectives}

LncRNAs may be regulators in the determination of the development of particular organs, rather than simply functioning as housekeeping genes. In contrast to other tissues, the brain expresses high levels of numerous lncRNAs, which are involved in neuro-development (94). Their deregulation may cause neurological disorders and brain tumors (95). As aforementioned, lncRNAs function as oncogenes or tumor suppressors by interacting with DNA, mRNA, ncRNA and proteins, and regulate the proliferation, migration, invasion, apoptosis, angiogenesis and stemness of glioma cells (Fig. 2). Recent studies of lncRNAs have highlighted their vital roles in the pathogenesis and progression of glioma. Furthermore, the sensitivity and reliability of RNA-based molecular technologies and tools to detect and target lncRNAs in glioma have improved (96). However, it should be noted that the current knowledge base regarding the biological roles of lncRNAs in glioma is primarily concerned with the identification and quantification of the expression levels of different lncRNAs and associated molecules in tumor and normal tissues. To date, the in vivo functions of a large proportion of the identified lncRNAs remain unknown. Therefore, a short-term goal would be to investigate the molecular, cellular and physiological functions of lncRNAs and their roles in pathogenesis of glioma, which will provide a foundation for developing novel medical therapies that target lncRNAs. High throughput technologies and massively parallel sequencing tools, in combination with bioinformatics methods, would assist in identifying lncRNA candidate targets whose dysregulation serves a pivotal role in the pathogenesis and progression of glioma. Genetically engineered mouse models would also be an indispensable tool to elucidate the functions and mechanisms of lncRNA genes in glioma in vivo. In summary, the identification of lncRNAs has revealed an additional facet of glioma tumorigenesis. Understanding the precise molecular mechanisms whereby lncRNAs function is important to advance lncRNA-based diagnosis, prognosis and therapeutic interventions against glioma.

\section{Acknowledgements}

The authors would like to thank Dr. Yunli Zhou (Neuroendocrine Unit, Massachusetts General Hospital, Harvard Medical School, Boston, MA, USA) for reviewing of the manuscript. The present research is supported by the Medical Research Fund for Young Scholars of the Sichuan Medical Association, Sichuan, China (grant no., Q16076) and the Natural Science Foundation of Southwest Medical University (grant no., 2016XNYD217).

\section{References}

1. Ponting CP, Oliver PL and Reik W: Evolution and functions of long noncoding RNAs. Cell 136: 629-641, 2009.

2. Kapranov P, Cheng J, Dike S, Nix DA, Duttagupta R, Willingham AT, Stadler PF, Hertel J, Hackermüller J, Hofacker IL, et al: RNA maps reveal new RNA classes and a possible function for pervasive transcription. Science 316: 1484-1488, 2007.

3. Schmitt AM and Chang HY: Long Noncoding RNAs in cancer pathways. Cancer Cell 29: 452-463, 2016. 
4. Hanahan D and Weinberg RA: Hallmarks of cancer: The next generation. Cell 144: 646-674, 2011.

5. Thomas J, Ohtsuka M, Pichler M and Ling H: MicroRNAs: Clinical relevance in colorectal cancer. Int J Mol Sci 16: 28063-28076, 2015.

6. Lee JS: The mutational landscape of hepatocellular carcinoma. Clin Mol Hepatol 21: 220-229, 2015.

7. Mattick JS and Makunin IV: Non-coding RNA. Hum Mol Genet 15 Spec No 1: R17-R29, 2006.

8. Han D, Wang M, Ma N, Xu Y, Jiang Y and Gao X: Long noncoding RNAs: Novel players in colorectal cancer. Cancer Lett 361: 13-21, 2015.

9. Rinn JL and Chang HY: Genome regulation by long noncoding RNAs. Annu Rev Biochem 81: 145-166, 2012.

10. Wang J, Song YX and Wang ZN: Non-coding RNAs in gastric cancer. Gene 560: 1-8, 2015.

11. Fatica A and Bozzoni I: Long non-coding RNAs: New players in cell differentiation and development. Nat Rev Genet 15: 7-21, 2014.

12. Lee JT: Epigenetic regulation by long noncoding RNAs. Science 338: 1435-1439, 2012.

13. Cabili MN, Trapnell C, Goff L, Koziol M, Tazon-Vega B, Regev A and Rinn JL: Integrative annotation of human large intergenic noncoding RNAs reveals global properties and specific subclasses. Genes Dev 25: 1915-1927, 2011.

14. Li R, Zhu H and Luo Y: Understanding the functions of long non-coding RNAs through their higher-order structures. Int J Mol Sci 17: E702, 2016.

15. Liu Y, Pan S, Liu L, Zhai X, Liu J, Wen J, Zhang Y, Chen J, Shen $\mathrm{H}$ and $\mathrm{Hu} \mathrm{Z}$ : A genetic variant in long non-coding RNA HULC contributes to risk of HBV-related hepatocellular carcinoma in a Chinese population. PLoS One 7: e35145, 2012.

16. Hashad D, Elbanna A, Ibrahim A and Khedr G: Evaluation of the role of circulating long non-coding RNA H19 as a promising novel biomarker in plasma of patients with gastric cancer. J Clin Lab Anal 30: 1100-1105, 2016.

17. Li H, Yu B, Li J, Su L, Yan M, Zhu Z and Liu B: Overexpression of lncRNA H19 enhances carcinogenesis and metastasis of gastric cancer. Oncotarget 5: 2318-2329, 2014.

18. Zhou X, Yin C, Dang Y, Ye F and Zhang G: Identification of the long non-coding RNA H19 in plasma as a novel biomarker for diagnosis of gastric cancer. Sci Rep 5: 11516, 2015.

19. Du Z, Fei T, Verhaak RG, Su Z, Zhang Y, Brown M, Chen Y and Liu XS: Integrative genomic analyses reveal clinically relevant long noncoding RNAs in human cancer. Nat Struct Mol Biol 20 908-913, 2013

20. Zhou M, Zhao H, Xu W, Bao S, Cheng L and Sun J: Discovery and validation of immune-associated long non-coding RNA biomarkers associated with clinically molecular subtype and prognosis in diffuse large B cell lymphoma. Mol Cancer 16: 16, 2017.

21. Hu Y, Chen HY, Yu CY, Xu J, Wang JL, Qian J, Zhang X and Fang JY: A long non-coding RNA signature to improve prognosis prediction of colorectal cancer. Oncotarget 5: 2230-2242, 2014

22. McCleland ML, Mesh K, Lorenzana E, Chopra VS, Segal E, Watanabe C, Haley B, Mayba O, Yaylaoglu M, Gnad F and Firestein R: CCAT1 is an enhancer-templated RNA that predicts BET sensitivity in colorectal cancer. J Clin Invest 126: 639-652, 2016.

23. Sun J, Chen X, Wang Z, Guo M, Shi H, Wang X, Cheng L and Zhou M: A potential prognostic long non-coding RNA signature to predict metastasis-free survival of breast cancer patients. Sci Rep 5: 16553, 2015.

24. Ostrom QT, Gittleman H, Farah P, Ondracek A, Chen Y, Wolinsky Y, Stroup NE, Kruchko C and Barnholtz-Sloan JS: CBTRUS statistical report: Primary brain and central nervous system tumors diagnosed in the united states in 2006-2010. Neuro Oncol 15 (Suppl 2): ii1-ii56, 2013.

25. Devaux Y, Zangrando J, Schroen B, Creemers EE, Pedrazzini T, Chang CP, Dorn GW II, Thum T and Heymans S; Cardioline network: Long noncoding RNAs in cardiac development and ageing. Nat Rev Cardiol 12: 415-425, 2015.

26. Takahashi H CPWgtnpfRtBBRC-, 2014

27. Ma L, Bajic VB and Zhang Z: On the classification of long non-coding RNAs. RNA Biol 10: 925-933, 2013.

28. Tsuiji H, Yoshimoto R, Hasegawa Y, Furuno M, Yoshida M and Nakagawa S: Competition between a noncoding exon and introns: Gomafu contains tandem UACUAAC repeats and associates with splicing factor-1. Genes Cells 16: 479-490, 2011.
29. Tripathi V, Ellis JD, Shen Z, Song DY, Pan Q, Watt AT, Freier SM Bennett CF, Sharma A, Bubulya PA, et al: The nuclear-retained noncoding RNA MALAT1 regulates alternative splicing by modulating SR splicing factor phosphorylation. Mol Cell 39: 925-938, 2010

30. Rintala-Maki ND and Sutherland LC: Identification and characterisation of a novel antisense non-coding RNA from the RBM5 gene locus. Gene 445: 7-16, 2009.

31. Lin D, Pestova TV, Hellen CU and Tiedge H: Translational control by a small RNA: Dendritic BC1 RNA targets the eukaryotic initiation factor 4A helicase mechanism. Mol Cell Biol 28 3008-3019, 2008

32. Shi X, Sun M, Liu H, Yao Y and Song Y: Long non-coding RNAs: A new frontier in the study of human diseases. Cancer Lett 339: 159-166, 2013.

33. Wang KC and Chang HY: Molecular mechanisms of long noncoding RNAs. Mol Cell 43: 904-914, 2011.

34. Raveh E, Matouk IJ, Gilon M and Hochberg A: The H19 Long non-coding RNA in cancer initiation, progression and metastasis-a proposed unifying theory. Mol Cancer 14: 184, 2015.

35. Hao Y, Crenshaw T, Moulton T, Newcomb E and Tycko B Tumour-suppressor activity of H19 RNA. Nature 365: 764-767, 1993.

36. Liu L, Yang J, Zhu X, Li D, Lv Z and Zhang X: Long noncoding RNA H19 competitively binds miR-17-5p to regulate YES1 expression in thyroid cancer. FEBS J 283: 2326-2339, 2016.

37. Han D, Gao X, Wang M, Qiao Y, Xu Y, Yang J, Dong N, He J, Sun Q, Lv G, et al: Long noncoding RNA H19 indicates a poor prognosis of colorectal cancer and promotes tumor growth by recruiting and binding to eIF4A3. Oncotarget 7: 22159-22173, 2016.

38. Matouk IJ, Raveh E, Abu-lail R, Mezan S, Gilon M, Gershtain E, Birman T, Gallula J, Schneider T, Barkali M, et al: Oncofetal H19 RNA promotes tumor metastasis. Biochim Biophys Acta 1843: 1414-1426, 2014

39. Jiang X, Yan Y, Hu M, Chen X, Wang Y, Dai Y, Wu D, Wang Y, Zhuang $\mathrm{Z}$ and $\mathrm{Xia} \mathrm{H}$ : Increased level of H19 long noncoding RNA promotes invasion, angiogenesis, and stemness of glioblastoma cells. J Neurosurg 124: 129-136, 2016.

40. Jia P, Cai H, Liu X, Chen J, Ma J, Wang P, Liu Y, Zheng J and Xue Y: Long non-coding RNA H19 regulates glioma angiogenesis and the biological behavior of glioma-associated endothelial cells by inhibiting microRNA-29a. Cancer Lett 381: 359-369, 2016.

41. Chen Y, Wu JJ, Lin XB, Bao Y, Chen ZH, Zhang CR, Cai Z, Zhou JY, Ding MH, Wu XJ, et al: Differential lncRNA expression profiles in recurrent gliomas compared with primary gliomas identified by microarray analysis. Int J Clin Exp Med 8: 5033-5043, 2015

42. Shi Y, Wang Y, Luan W, Wang P, Tao T, Zhang J, Qian J, Liu N and You Y: Long non-coding RNA H19 promotes glioma cell invasion by deriving miR-675. PLoS One 9: e86295, 2014.

43. Poirier F, Chan C, Timmons P, Robertson EJ, Evans MJ and Rigby PW: The murine H19 gene is activated during embryonic stem cell differentiation in vitro and at the time of implantation in the developing embryo. Development 113: 1105-1114, 1991.

44. Venkatraman A, He XC, Thorvaldsen JL, Sugimura R, Perry JM, Tao F, Zhao M, Christenson MK, Sanchez R, Yu JY, et al: Maternal imprinting at the H19-Igf2 locus maintains adult haematopoietic stem cell quiescence. Nature 500: 345-349, 2013.

45. Yin Y, Wang H, Liu K, Wang F, Ye X, Liu M, Xiang R, Liu N and Liu L: Knockdown of H19 enhances differentiation capacity to epidermis of parthenogenetic embryonic stem cells. Curr Mol Med 14: 737-748, 2014.

46. Jiang X, Yan Y, Hu M, Chen X, Wang Y, Dai Y, Wu D, Wang Y, Zhuang $\mathrm{Z}$ and $\mathrm{Xia} \mathrm{H}$ : Increased level of H19 long noncoding RNA promotes invasion, angiogenesis, and stemness of glioblastoma cells. J Neurosurg 2016: 129-136, 2016.

47. Li W, Jiang P, Sun X, Xu S, Ma X and Zhan R: Suppressing H19 modulates tumorigenicity and stemness in U251 and U87MG glioma cells. Cell Mol Neurobiol 36: 1219-1227, 2016.

48. Jiang P, Wang P, Sun X, Yuan Z, Zhan R, Ma X and Li W: Knockdown of long noncoding RNA H19 sensitizes human glioma cells to temozolomide therapy. Onco Targets Ther 9: 3501-3509, 2016

49. Wang J, Pan Y, Wu J, Zhang C, Huang Y, Zhao R, Cheng G, Liu J, Qin C, Shao P, et al: The association between abnormal long Noncoding RNA MALAT-1 expression and cancer lymph node metastasis: A meta-analysis. Biomed Res Int 2016: 1823482, 2016. 
50. Ji P, Diederichs S, Wang W, Böing S, Metzger R, Schneider PM, Tidow N, Brandt B, Buerger H, Bulk E, et al: MALAT-1, a novel noncoding RNA, and thymosin beta4 predict metastasis and survival in early-stage non-small cell lung cancer. Oncogene 22: 8031-8041, 2003.

51. Zhou Y, Xu X, Lv H, Wen Q, Li J, Tan L, Li J and Sheng X: The Long Noncoding RNA MALAT-1 is highly expressed in ovarian cancer and induces cell growth and migration. PLoS One 11: e0155250, 2016

52. Han T, Jiao F, Hu H, Yuan C, Wang L, Jin ZL, Song WF and Wang LW: EZH2 promotes cell migration and invasion but not alters cell proliferation by suppressing E-cadherin, partly through association with MALAT-1 in pancreatic cancer. Oncotarget 7: 11194-11207, 2016.

53. Tano K, Mizuno R, Okada T, Rakwal R, Shibato J, Masuo Y, Ijiri K and Akimitsu N: MALAT-1 enhances cell motility of lung adenocarcinoma cells by influencing the expression of motility-related genes. FEBS Lett 584: 4575-4580, 2010.

54. Liu S, Song L, Zeng S and Zhang L: MALAT1-miR-124-RBG2 axis is involved in growth and invasion of HR-HPV-positive cervical cancer cells. Tumour Biol 37: 633-640, 2016.

55. Ma KX, Wang HJ, Li XR, Li T, Su G, Yang P and Wu JW: Long noncoding RNA MALAT1 associates with the malignant status and poor prognosis in glioma. Tumour Biol 36: 3355-3359, 2015.

56. Xiang J, Guo S, Jiang S, Xu Y, Li J, Li L and Xiang J: Silencing of Long Non-coding RNA MALAT1 promotes apoptosis of glioma cells. J Korean Med Sci 31: 688-694, 2016.

57. Vassallo I,Zinn P, Lai M, Rajakannu P, Hamou MF and Hegi ME: WIF1 re-expression in glioblastoma inhibits migration through attenuation of non-canonical WNT signaling by downregulating the IncRNA MALAT1. Oncogene 35: 12-21, 2016.

58. Ma J, Wang P, Yao Y, Liu Y, Li Z, Liu X, Li Z, Zhao X, Xi Z, Teng H, et al: Knockdown of long non-coding RNA MALAT1 increases the blood-tumor barrier permeability by up-regulating miR-140. Biochim Biophys Acta 1859: 324-338, 2016.

59. Han Y, Wu Z, Wu T, Huang Y, Cheng Z, Li X, Sun T, Xie X, Zhou Y and Du Z: Tumor-suppressive function of long noncoding RNA MALAT1 in glioma cells by downregulation of MMP2 and inactivation of ERK/MAPK signaling. Cell Death Dis 7: e2123, 2016.

60. Cai B, Song XQ, Cai JP and Zhang S: HOTAIR: A cancer-related long non-coding RNA. Neoplasma 61: 379-391, 2014.

61. Xue X, Yang YA, Zhang A, Fong KW, Kim J, Song B, Li S, Zhao JC and Yu J: LncRNA HOTAIR enhances ER signaling and confers tamoxifen resistance in breast cancer. Oncogene 35: 2746-55, 2016.

62. Fu WM, Zhu X, Wang WM, Lu YF, Hu BG, Wang H, Liang WC, Wang SS, Ko CH, Waye MM, et al: Hotair mediates hepatocarcinogenesis through suppressing miRNA-218 expression and activating P14 and P16 signaling. J Hepatol 63: 886-895, 2015.

63. Zhang JX, Han L, Bao ZS, Wang YY, Chen LY, Yan W, Yu SZ $\mathrm{Pu}$ PY, Liu N, You YP, et al: HOTAIR, a cell cycle-associated long noncoding RNA and a strong predictor of survival, is preferentially expressed in classical and mesenchymal glioma. Neuro Oncol 15: 1595-1603, 2013.

64. Zhou X, Ren Y, Zhang J, Zhang C, Zhang K, Han L, Kong L, Wei J, Chen L, Yang J, et al: HOTAIR is a therapeutic target in glioblastoma. Oncotarget 6: 8353-8365, 2015

65. Fang K, Liu P, Dong S, Guo Y, Cui X, Zhu X, Li X, Jiang L, Liu T and Wu Y: Magnetofection based on superparamagnetic iron oxide nanoparticle-mediated low lncRNA HOTAIR expression decreases the proliferation and invasion of glioma stem cells. Int J Oncol 49: 509-518, 2016.

66. Liz J and Esteller M: IncRNAs and microRNAs with a role in cancer development. Biochim Biophys Acta 1859: 169-176, 2016

67. Ke J, Yao YL, Zheng J, Wang P, Liu YH, Ma J, Li Z, Liu XB, Li ZQ, Wang ZH and Xue YX: Knockdown of long non-coding RNA HOTAIR inhibits malignant biological behaviors of human glioma cells via modulation of miR-326. Oncotarget 6 : 21934-21949, 2015.

68. Yang B, Wei ZY, Wang BQ, Yang HC, Wang JY and Bu XY: Down-regulation of the long noncoding RNA-HOX transcript antisense intergenic RNA inhibits the occurrence and progression of glioma. J Cell Biochem 119: 2278-2287, 2018.

69. Bian EB, Ma CC, He XJ, Wang C, Zong G, Wang HL and Zhao B: Epigenetic modification of miR-141 regulates SKA2 by an endogenous 'sponge' HOTAIR in glioma. Oncotarget 7: 30610-30625, 2016.
70. Wang G, Li Z, Tian N, Han L, Fu Y, Guo Z and Tian Y: miR-148b-3p inhibits malignant biological behaviors of human glioma cells induced by high HOTAIR expression. Oncol Lett 12: 879-886, 2016.

71. Graham LD, Pedersen SK, Brown GS, Ho T, Kassir Z, Moynihan AT, Vizgoft EK, Dunne R, Pimlott L, Young GP, et al: Colorectal neoplasia differentially expressed (CRNDE), a novel gene with elevated expression in colorectal adenomas and adenocarcinomas. Genes Cancer 2: 829-840, 2011.

72. Ellis BC, Molloy PL and Graham LD: CRNDE: A long non-coding RNA involved in cancer, neurobiology, and development. Front Genet 3: 270, 2012.

73. Kiang K, Zhang XQ and Leung, GK: Long non-coding RNAs: The key players in glioma pathogenesis. Cancers (Basel) 7: 1406-1424, 2015.

74. Zhang X, Sun S, Pu JK, Tsang AC, Lee D, Man VO, Lui WM, Wong ST and Leung GK: Long non-coding RNA expression profiles predict clinical phenotypes in glioma. Neurobiol Dis 48: $1-8,2012$

75. Zheng J, Liu X, Wang P, Xue Y, Ma J, Qu C and Liu Y: CRNDE promotes malignant progression of glioma by attenuating miR-384/PIWIL4/STAT3 axis. Mol Ther 24: 1199-1215, 2016.

76. Wang Y, Wang Y, Li J, Zhang Y, Yin H and Han B: CRNDE, a long-noncoding RNA, promotes glioma cell growth and invasion through mTOR signaling. Cancer Lett 367: 122-128, 2015.

77. Palmieri G, Paliogiannis P, Sini MC, Manca A, Palomba G, Doneddu V, Tanda F, Pascale MR and Cossu A: Long non-coding RNA CASC2 in human cancer. Crit Rev Oncol Hematol 111: 31-38, 2017.

78. Wang P, Liu YH, Yao YL, Li Z, Li ZQ, Ma J and Xue YX: Long non-coding RNA CASC2 suppresses malignancy in human gliomas by miR-21. Cell Signal 27: 275-282, 2015.

79. Liao Y, Shen L, Zhao H, Liu Q, Fu J, Guo Y, Peng R and Cheng L: LncRNA CASC2 interacts with miR-181a to modulate glioma growth and resistance to TMZ through PTEN pathway. J Cell Biochem 118: 1889-1899, 2017.

80. Zhou Y, Zhang X and Klibanski A: MEG3 noncoding RNA: A tumor suppressor. J Mol Endocrinol 48: R45-R53, 2012.

81. Zhang X, Zhou Y, Mehta KR, Danila DC, Scolavino S, Johnson SR and Klibanski A: A pituitary-derived MEG3 isoform functions as a growth suppressor in tumor cells. J Clin Endocrinol Metab 88: 5119-5126, 2003.

82. Balci T, Yilmaz Susluer S, Kayabasi C, Ozmen Yelken B, Biray Avci C and Gunduz C: Analysis of dysregulated long non-coding RNA expressions in glioblastoma cells. Gene 590: 120-122, 2016.

83. Wang P, Ren Z and Sun P: Overexpression of the long non-coding RNA MEG3 impairs in vitro glioma cell proliferation. J Cell Biochem 113: 1868-1874, 2012.

84. Liu Q, Sun S, Yu W, Jiang J, Zhuo F, Qiu G, Xu S and Jiang X: Altered expression of long non-coding RNAs during genotoxic stress-induced cell death in human glioma cells. J Neurooncol 122: 283-292, 2015.

85. Zhang L, Liang X and Li Y: Long non-coding RNA MEG3 inhibits cell growth of gliomas by targeting miR-93 and inactivating PI3K/AKT pathway. Oncol Rep 38: 2408-2416, 2017.

86. Reon BJ, Anaya J, Zhang Y, Mandell J, Purow B, Abounader R and Dutta A: Expression of lncRNAs in low-grade gliomas and glioblastoma multiforme: An In silico analysis. PLoS Med 13: e1002192, 2016.

87. Zhang XQ, Kiang KM, Wang YC, Pu JK, Ho A, Cheng SY, Lee D, Zhang PD, Chen JJ, Lui WM, et al: IDH1 mutation-associated long non-coding RNA expression profile changes in glioma. J Neurooncol 125: 253-263, 2015.

88. Wang W, Yang F, Zhang L, Chen J, Zhao Z, Wang H, Wu F, Liang T, Yan X, Li J, et al: LncRNA profile study reveals four-lncRNA signature associated with the prognosis of patients with anaplastic gliomas. Oncotarget 7: 77225-77236, 2016.

89. Evans JR, Feng FY and Chinnaiyan AM: The bright side of dark matter: IncRNAs in cancer. J Clin Invest 126: 2775-2782, 2016.

90. Arun G, Diermeier S, Akerman M, Chang KC, Wilkinson JE, Hearn S, Kim Y, MacLeod AR, Krainer AR, Norton L, et al: Differentiation of mammary tumors and reduction in metastasis upon Malat1 lncRNA loss. Genes Dev 30: 34-51, 2016.

91. Amit D, Matouk IJ, Lavon I, Birman T, Galula J, Abu-Lail R, Schneider T, Siegal T, Hochberg A and Fellig Y: Transcriptional targeting of glioblastoma by diphtheria toxin-A driven by both H19 and IGF2-P4 promoters. Int J Clin Exp Med 5: 124-135, 2012. 
92. Pastori C, Kapranov P, Penas C, Peschansky V, Volmar CH, Sarkaria JN, Bregy A, Komotar R, St Laurent G, Ayad NG and Wahlestedt C: The Bromodomain protein BRD4 controls HOTAIR, a long noncoding RNA essential for glioblastoma proliferation. Proc Natl Acad Sci USA 112: 8326-8331, 2015.

93. García-Claver A, Lorente M, Mur P, Campos-Martín Y, Mollejo M, Velasco G and Meléndez B: Gene expression changes associated with erlotinib response in glioma cell lines. Eur J Cancer 49: 1641-1653, 2013.

94. Shi C, Zhang L and Qin C: Long non-coding RNAs in brain development, synaptic biology, and Alzheimer's disease. Brain Res Bull 132: 160-169, 2017.

95. Subhramanyam CS and Hu Q: Non-coding rna in brain development and disorder. Curr Med Chem 24: 1983-1997, 2017.

96. Hassan A, Mosley J, Singh S and Zinn PO: A comprehensive review of genomics and noncoding RNA in gliomas. Top Magn Reson Imaging 26: 3-14, 2017.

97. Hu L, Lv QL, Chen SH, Sun B, Qu Q, Cheng L, Guo Y, Zhou HH and Fan L: Up-Regulation of long non-coding RNA AB073614 predicts a poor prognosis in patients with glioma. Int J Environ Res Public Health 13: 433, 2016.

98.Zhu Y, Zhang X, Qi L, Cai Y, Yang P, Xuan G and Jiang Y: HULC long noncoding RNA silencing suppresses angiogenesis by regulating ESM-1 via the $\mathrm{PI} 3 \mathrm{~K} / \mathrm{Akt} / \mathrm{mTOR}$ signaling pathway in human gliomas. Oncotarget 7: 14429-14440, 2016.

99. He C, Jiang B, Ma J and Li Q: Aberrant NEAT1 expression is associated with clinical outcome in high grade glioma patients. APMIS 124: 169-74, 2016.
100. Zhen L, Yun-Hui L, Hong-Yu D, Jun M and Yi-Long Y: Long noncoding RNA NEAT1 promotes glioma pathogenesis by regulating miR-449b-5p/c-Met axis. Tumour Biol 37: 673-683, 2016.

101. Guo H, Wu L, Yang Q, Ye M and Zhu X: Functional linc-POU3F3 is overexpressed and contributes to tumorigenesis in glioma. Gene 554: 114-119, 2015.

102. Liu H, Lv Z and Guo E: Knockdown of long noncoding RNA SPRY4-IT1 suppresses glioma cell proliferation, metastasis and epithelial-mesenchymal transition. Int J Clin Exp Pathol 8: 9140-9146, 2015.

103. Yao Y, Ma J, Xue Y, Wang P, Li Z, Liu J, Chen L, Xi Z, Teng H, Wang Z, et al: Knockdown of long non-coding RNA XIST exerts tumor-suppressive functions in human glioblastoma stem cells by up-regulating miR-152. Cancer Lett 359: 75-86, 2015.

104. Yao J, Zhou B, Zhang J, Geng P, Liu K, Zhu Y and Zhu W: A new tumor suppressor LncRNA ADAMTS9-AS2 is regulated by DNMT1 and inhibits migration of glioma cells. Tumour Biol 35: 7935-7944, 2014

105. Li J, Bian EB, He XJ, Ma CC, Zong G, Wang HL and Zhao B Epigenetic repression of long non-coding RNA MEG3 mediated by DNMT1 represses the p53 pathway in gliomas. Int J Oncol 48: 723-733, 2016

106. Qin X, Yao J, Geng P, Fu X, Xue J and Zhang Z: LncRNA TSLC1-AS1 is a novel tumor suppressor in glioma. Int J Clin Exp Pathol 7: 3065-3072, 2014.

This work is licensed under a Creative Commons Attribution-NonCommercial-NoDerivatives 4.0 International (CC BY-NC-ND 4.0) License. 